\title{
analyse de la stabilité des murs de soutènement par le calcul à la rupture
}

\author{
par \\ M. Mommessin \\ Assistant, I.U.T, de Limoges, département Génie Civil \\ et \\ R. Nègre \\ Professeur, Université Scientifique et Médicale de Grenoble
}

\section{Introduction}

L'analyse de la stabilité des murs poids de soutènement a été l'objet de nombreuses études, mais pratiquement toutes les méthodes de calcul proposées jusqu'à présent étudient séparément l'action des terres sur la paroi et la stabilité de la fondation de l'ouvrage, perdant de ce fait toute signification du point de vue mécanique.

La théorie du calcul à la rupture, récemment mise en forme par Salençon (1978), à partir des travaux de Drucker (1953) et Radenkovic (1961), permet une analyse de la stabilité de tels ouvrages par une méthode rigoureuse du point de vue mécanique. Développée sous la seule hypothèse de convexité du critère de résistance du matériau constitutif de l'ouvrage, cette théorie permet, dans une géométrie fixée, de définir l'ensemble des chargements pour lesquels il ne sera pas possible de réaliser l'équilibre de l'ouvrage sans violer le critère de résistance et ceci quelles que soient les autres propriétés du matériau.

La comparaison des résultats ainsi obtenus avec ceux des méthodes classiques de calcul des murs de soutènement permet donc une meilleure interprétation de ces dernières.

\section{Présentation du calcul à la rupture}

\subsection{Présentation du calcul à la rupture}

Nous nous contenterons de rappeler ici les hypothèses et les principaux résultats du calcul à la rupture, thèorie largement présentée par ailleurs (Salençon 1978).

On considère un système mécanique $\Omega$ que l'on étudie dans sa géométrie initiale, c'est-à-dire qu'aucun changement de géométrie ne sera pris en compte avant la rupture possible du système.
On suppose qu'en chaque point du système $\Omega$, la capacité de résistance du matériau constitutif est définie par un critère convexe f, fonction scalaire de $g$, tenseur des contraintes en ce point. Le critère $f$ définit un domaine $(G)$, convexe dans l'espace des contraintes.

On suppose que, le système $\Omega$ étant soumis à un processus de chargement $Q$ dépendant de $n$ paramètres $Q_{1}, \ldots, Q_{n}$ il est possible de leur associer dans l'expression des forces extérieures les variables $q_{1}, \ldots . . q_{n}$ paramétres cinématiques associés, de telle sorte que, pour tout mécanisme de déformation ( $\underline{U}, \underline{\text { d}})$, avec $U$ champ de vitesses de déplacement et d tenseur des vitesses de déformation, cinématiquement admissible (C.A.), la puissance des efforts extérieurs se mette sous la forme :

$$
P_{e}(U)=Q_{1} \cdot q_{1}=\underline{Q} \cdot \underline{q}(\underline{U}) \text {. }
$$

II est clair que pour qu'un chargement $Q$ puisse ètre supporté par le système $\Omega$, il est nécessaire que l'on puisse trouver au moins un champ de contraintes $\sigma$ en équilibre avec $Q$ et respectant le critère. Autrement dit, on a :

$\underline{\sigma}$ supporte $\underline{Q} \Longrightarrow \underline{\underline{\sigma}}$ tel que $:\left\{\begin{array}{l}\underline{\underline{\sigma}} \text { équilibre } \underline{Q} \\ \underline{\underline{\sigma}} \in(G), \forall M \in \Omega .\end{array}\right.$

Les conditions (2) énoncées ci-dessus montre que $Q$ doit appartenir à un domaine $(\mathrm{K})$, convexe en raison de la convexité du critère. (K) est appelé convexe des chargements potentiellement supportables.

On peut donc affirmer que le système ne saurait supporter, en géométrie initiale, un chargement extérieur à (K). Par contre, la question de savoir si le système supportera effectivement tous les chargements intérieurs à $(K)$ ne saurait 'être traitée sans informations complémentaires sur le comportement du matériau.

La frontière $F(K)$ du convexe des chargements potentiellement supportables par le système peut être 


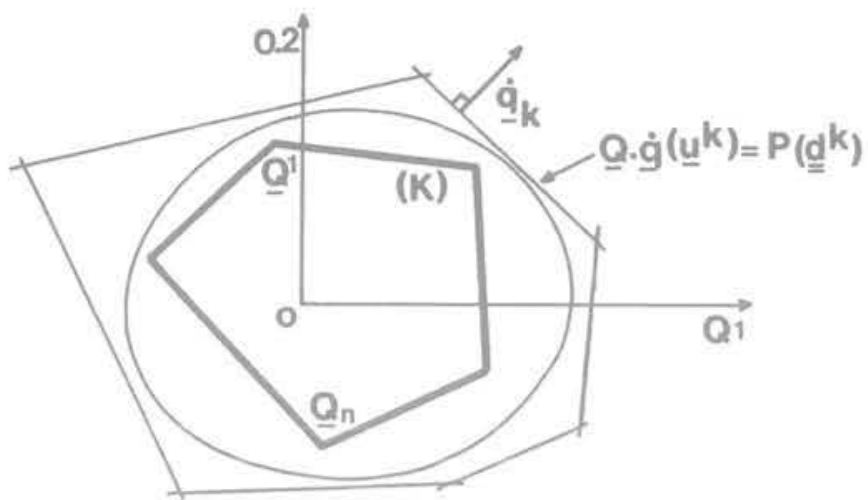

Fig. 1 Approches intérieur et extérieur de la frontière du convexe $(K)$ (chargement à 2 paramètres)

déterminée par deux méthodes, mathématiquement duales l'une de l'autre (fig. 1):

- approche par l'intérieur par la construction de champs de contraintes en équilibre et respectant le critère de résistance,

- approche par l'extérieur, par la construction de mécanismes de déformation cinématiquement admissibles et le calcul dans ceux-ci de la puissance dissipable $P(d)$ (terminologie introduite par Coussy 1978). En effet, on a alors: $\underline{Q} \cdot \underline{q} \leqslant P(d)$.

2.2 Application du calcul à la rupture à l'étude de la tenue d'un ouvrage

2.2.1 Tenue d'un ouvrage sous un chargement donné

Lors de l'étude de la tenue d'un ouvrage, le problème qui se pose est le suivant:

Étant donné un ouvrage dont on connait la capacité de résistance du matériau en chaque point,

Étant donné le chargement auquel est soumis cet ouvrage,

quelle est la "sécuritén de cet ouvrage vis-à-vis de la ruine?

Désignant par $\underline{Q}$ le chargernent de l'ouvrage, le problème posé revient, du point de vue calcul à la rupture, à situer $Q$ par rapport à la frontière du convexe $(K)$ des chargements potentiellement supportables par l'ouvrage.

2.2.2 Coefficients multiplicateurs de charge attachés à un chargement donné

Considérons un trajet de charge linéaire défini par une direction $A$ ( $A$ vecteur unitaire). Soit $Q$, le chargement extrême pour ce trajet de charge (fig. 2).

On peut définir des coefficients $F_{i}$ tels que:

$$
Q_{t_{1}}=F_{i} \cdot Q_{i}, i=1, n
$$

Ces coefficients $F_{\text {i }}$ sont les coefficients multiplicateurs de charge attachés au chargement $\underline{Q}$ dans la direction $A$ et l'on peut définir un coefficient $F$, tel que :

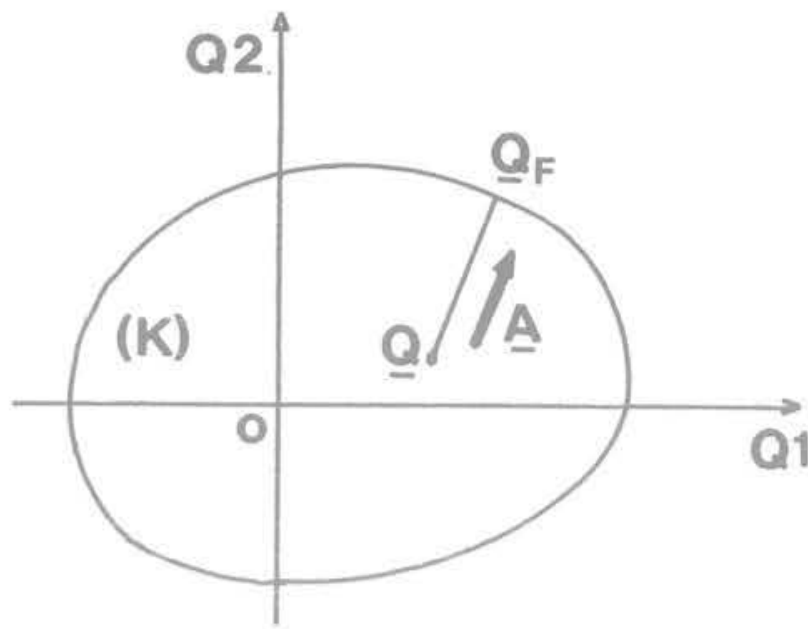

Fig. 2 Chargement linéaire dans la direction de charge $A$, à partir de $Q$

$$
\underline{Q}_{1}=\underline{Q}+F \cdot \underline{A}
$$

Si $F$ est positif, $Q$ est intérieur à $(K)$, sinon il est extérieur.

Les coefficients $F_{i}$ se déduisent de $F$ par :

$$
F_{1}=F \cdot a_{i} / Q_{1}+1 \text {, si } Q_{i} \neq 0
$$

$\left(a_{i}\right.$ composantes de $\left.\underline{A}\right)$

On peut donc définir les coefficients multiplicateurs de charge $F_{i}$ à partir d'un coefficient unique $F$, tel que si $\mathrm{F}$ est négatif, la rupture de l'ouvrage sous le chargement $Q$ est certaine. Si $F$ est positif, les coefficients $F_{i}$ permettent de savoir pour quelle augmentation de charge dans la direction A la rupture sera certaine. $F$ représente la mesure algébrique de $Q_{i}-\underline{Q}, F$ peut donc être qualifié d'accroissement ultime de charge dans la direction $\underline{A}$ (accroissement potentiellement ultime).

\subsubsection{Approche statique et cinématique de $\mathrm{F}$}

\section{Approche statique}

II est évident que si l'on a trouvé un champ de contraintes $\underline{\sigma}$, appartenant à (G), et équilibrant $Q_{\lambda}=Q+\lambda \cdot A, \lambda$ constitue alors une approximation par défaut de $F$.

\section{Approche cinématique}

Le chargement extrême $Q_{t}$ possède la propriété suivante :

$\forall \underline{\text { d }}$ cinématiquement admissible, $Q, \cdot q(\underline{\mathrm{U}}) \leqslant P(\underline{\underline{d}}) \quad(6)$.

En remplaçant $Q$, par $\underline{Q}+F \cdot \underline{A}$, on a donc :

$\mathrm{F} \leqslant \operatorname{lnf}\{(\mathrm{P}(\underline{\mathrm{d}})-\underline{Q} \cdot \underline{q}(\underline{\mathrm{U}}))\}$

$$
(\underline{A} \cdot \underline{q}(\underline{U})) \mid \underline{d} C \cdot A \cdot: \underline{A} \cdot \underline{q}(\underline{U})>0\}
$$

La mise en évidence d'un mécanisme de déformation $(\underline{U}, \underline{d})$, cinématiquement admissible, et tel que $A \cdot q(\underline{U})$ soit positif conduit donc à une approximation de $F$. Le majorant ainsi obtenu gardant la même signification que le coefficient exact vis-à-vis de la possibilité de ruine de l'ouvrage étudié.

\subsubsection{Trajets de charge particuliers}

\section{- Chargement radial.}

Considérons un trajet de charge radial (fig. 3), on peut 


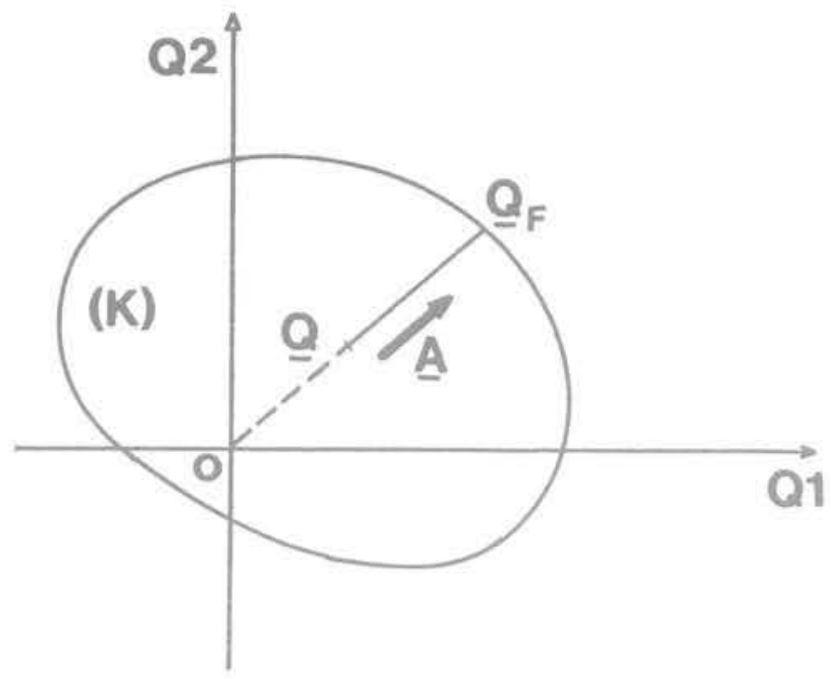

Fig. 3 Trajet de charge radial (chargement à 2 paramètres)

alors poser $A=Q /|Q|$. La formule (4) devient alors $\underline{Q}_{1}=(1+F /|\underline{Q}|) \cdot \underline{Q}$, et l'on a alors :

$$
\begin{gathered}
\forall i, \quad i=1, n, \\
F_{1}=F /|\underline{Q}|+1 \leqslant \operatorname{lnf}\{P(\underline{d}) /(\underline{Q} \\
\cdot \underline{q}(\underline{U})) \mid \underline{d} C . A .: \underline{Q} \cdot \underline{q}(\underline{U})>0\}
\end{gathered}
$$

\section{- Chargement parallèle à un axe :}

Considérons un trajet de charge parallèle à l'axe $Q_{p}$ (fig. 4). On a $Q_{t}$ égale à $Q_{i}$ si i est différent de $p$ et $Q_{i_{p}}=F_{p} \cdot Q_{p}$. Soit :

$$
\begin{aligned}
& F_{p} \leqslant \operatorname{lnf}\{(P(\underline{d})-\underline{Q} \cdot \underline{q}(\underline{U}))\} \\
& \left.\left(Q_{p} \cdot \dot{q}_{p}(\underline{U})\right)+1 \mid \underline{\underline{d} C} \cdot A \cdot: \underline{Q}_{P} \cdot \underline{q}_{P}>0\right\} \\
& F_{i}=1 \text { si } i \neq p \text {. }
\end{aligned}
$$

\section{Application à l'analyse de la stabilité d'un mur poids}

\subsection{Présentation de l'ouvrage étudié}

\subsubsection{La géométrie}

On considère un massif de sol de hauteur $\mathrm{H}$, à surface libre inclinée de $\beta$ sur l'horizontale, soutenu par un mur-poids (fig. 5). La base du mur est inclinée de $\alpha$ par rapport à I'horizontale et a pour largeur B; son parement est incliné de $\lambda$ par rapport à la verticale. La surface libre du sol en aval du mur est horizontale. Lorsqu'il existe une nappe phréatique dans le remblai, sur une hauteur $\mathrm{H}_{w}$, on admet qu'elle est en équilibre. On suppose que l'ouvrage à une longueur infinie.

\subsubsection{Le chargement}

Outre les forces dues à la gravité. l'ouvrage est soumis à des surcharges extérieures $p_{1}$ et $p_{2}$, respectivement à la surface libre du remblai et du sol d'assise. On raisonne en contraintes effectives, l'eau est donc considérée comme un agent extérieur. Soit $\gamma_{w}$ le poids volumique de l'eau. Notons $W_{b}$ le poids du mur, $\gamma_{1}$ le poids volumique du sol dans la partie hors d'eau du remblai, $\gamma_{1}^{\prime}$ le poids volumique déjaugé du remblai et $\gamma_{2}^{\prime}$ celui du sol d'assise.

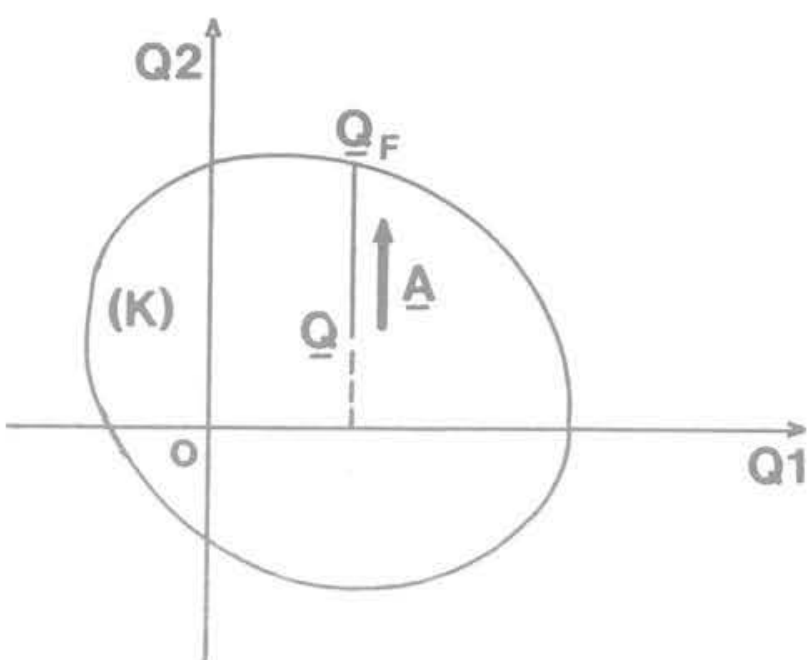

Fig. 4 Trajet de charge parallèle à l'axe $Q_{2}$ (chargement à 2 paramètres)

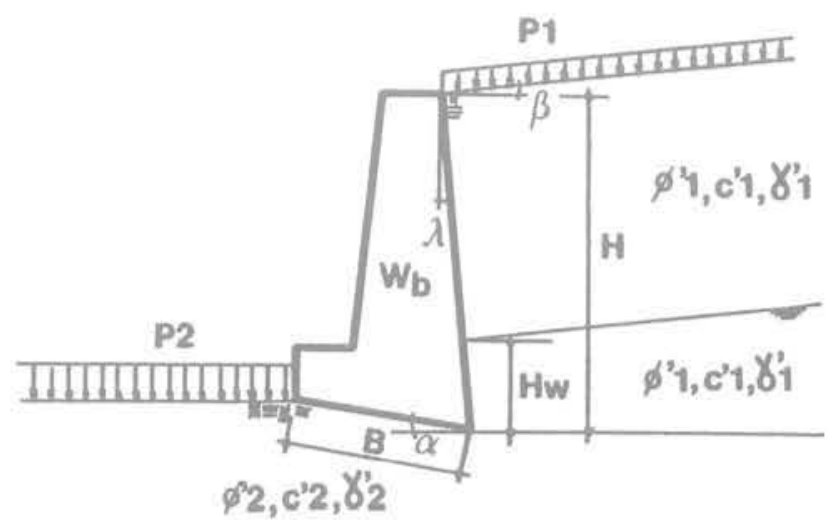

Fig. 5 L'ouvrage étudié

On peut choisir comme paramètres de chargements adimensionnels:

$$
\begin{gathered}
Q B=W_{b} /\left(C_{2}^{\prime} \cdot B\right), \quad Q S_{1}=\gamma_{1} \cdot H / C_{2}^{\prime}, \\
Q S_{1}^{\prime}=\gamma_{1}^{\prime} \cdot H / C_{2}^{\prime}, \quad Q S_{2}=\gamma_{2}^{\prime} \cdot H / C_{2,}^{\prime} \\
Q W=\gamma_{w} \cdot H / C_{2}^{\prime}, \quad Q P_{1}=p_{1} / C_{2}^{\prime} . \\
Q P_{2}=p_{2} / C_{2}^{\prime} .
\end{gathered}
$$

$\mathrm{C}_{2}^{\prime}$ étant la cohésion du sol d'assise.

\subsubsection{Les critères de résistance}

Le mur est supposé indéformable.

Le remblai est supposé obéir au critère de Coulomb. La cohésion et l'angle de frottement interne sont notés $C_{i}^{\prime}$ et $\phi_{i}^{\prime}$. Le sol d'assise obéit au critère de Coulomb de cohésion $C_{2}^{\prime}$ et d'angle de frottement interne $\phi_{2}^{\prime}$, ou au critère de Tresca de cohésion $\mathrm{C}_{2}^{\prime}$ $\left(\phi_{2}^{\prime}=0\right)$ si le matériau est une argile saturée et que l'on se préoccupe du comportement à court terme.

Les interfaces remblai-mur et sol d'assise-mur sont à frottement de Coulomb, ou de Tresca sans résistance à la traction pour l'interface sol d'assise-mur, si le critère régissant le sol d'assise est celui de Tresca. L'interface remblai-sol d'assise est une interface collée, la rupture aura donc lieu soit dans le sol d'assise soit dans le remblai.

L'ouvrage ayant une largeur infinie et compte tenu du chargement on peut étudier le problème en 


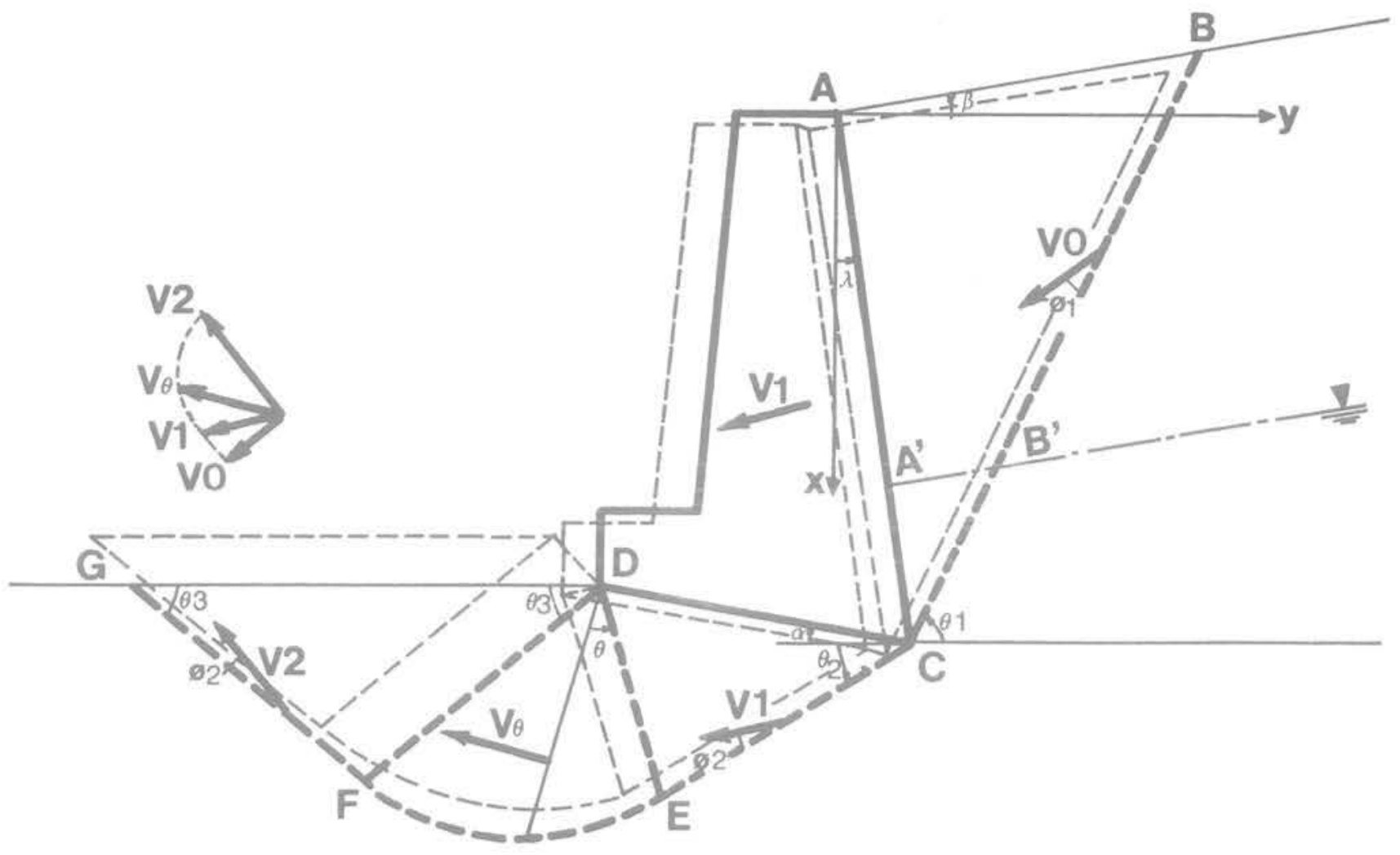

Fig. 6 Approche cinématique - Le mécanisme envisagé

déformation plane. L'expression des critères et des fonctions d'appui correspondantes est donnée dans Mommessin (1981).

\subsection{Approche extérieure du convexe}

\subsubsection{Le mécanisme considéré}

De nombreuses expériences réalisées à l'Université de Grenoble sur des modèles réduits de murs poids de formes diverses ont permis de constater qu'à la rupture il existe toujours un coin de sol solidaire de la base du mur (Martial - 1972). Aussi, envisageonsnous le mécanisme représenté sur la figure 6 .

Le bloc $A B C$ rigide se déplace en translation à une vitesse $\underline{V}_{0}$. Le mur ainsi que le coin rigide $D C E_{\text {, }}$ solidaire de sa base, se déplace en translation à une vitesse $V_{1}$. Le bloc DEF, limité par l'arc de spirale logarithmique EF (angle $\phi_{2}^{\prime}$ ) se déforme, sa vitesse est $V_{t}=V_{1} \cdot \exp \left(\theta \cdot \operatorname{tg} \phi_{2}^{\prime}\right)$. DFG est un bloc rigide qui se déplace en translation à la vitesse $\mathrm{V}_{2}$. $\mathrm{V}_{0}$ est incliné de $\phi_{1}^{\prime}$ sur la ligne de discontinuité $\mathrm{BC}$. La vitesse relative $\underline{V}_{1}-\underline{V}_{0}$ le long de $A C$ est inclinée de $\phi_{1}^{\prime}$ sur cette ligne. $\underline{V}_{1}$ fait un angle $\phi_{2}^{\prime}$ avec CE. II n'y a pas de glissement le long de DE et de DF.

EF étant un arc de spirale logarithmique d'angle $\phi_{2}^{\prime}$, la vitesse le long de cette ligne, perpendiculaire au rayon vecteur est inclinée de $\phi_{2}^{\prime}$ sur la discontinuité. $\underline{\mathrm{V}}_{2}$ fait un angle $\phi_{2}^{\prime}$ avec $\mathrm{FG}$.

On a : angle $(\mathrm{CK}, \mathrm{CB})=\theta_{1}$ angle $(C D, C E)=\theta_{2}-\alpha$ butée).
Afin d'assurer la continuité des lignes de glissement en $E$ et $F$, et de ce fait le non-glissement le long de $\mathrm{DE}$ et $\mathrm{DF}$, on est conduit à prendre

$$
(E C, E D)=\pi / 2-\phi_{2}^{\prime}, \quad(F D, F G)=\pi / 2+\phi_{2}^{\prime} .
$$

Le champ de vitesses de déformation $\mathrm{d}$, dérivant du champ de vitesses de déplacement décrit ci-dessus est cinématiquement admissible. De plus, il conduit à une valeur finie de la puissance dissipable.

Le détail des calcuis de la puissance dissipable et de la puissance des forces extérieures est donné dans Mommessin (1981).

\subsubsection{Déroulement pratique du calcul}

Soit F laccroissement ultime de charge dans la direction de charge $A$ (vecteur de composantes $a_{B}$. $\left.a_{S_{1}}, a_{S_{2}}, a_{S_{1}}, a_{W}, a_{p_{1}}, a_{p_{2}}\right)$, associé au chargement $Q$ $\left(Q_{B_{1}}, Q_{S_{1}}, Q_{S_{2}}, Q_{S_{1}}, Q_{p_{1}}, Q_{p_{2}}\right)$ donné. Quel que soit la valeur des angles $\theta_{1}$ et $\theta_{2}$, l'expression :

$[(P(\underline{d})-\underline{Q} \cdot \underline{q}) /(\underline{A} \cdot \underline{q}) / A \cdot \underline{q}>0]=F^{+}\left(\theta_{1}, \theta_{2}\right)$

est un majorant de $F$.

La minimisation de $\mathrm{F}^{+}$, fonction des deux variables $\theta_{1}$ et $\theta_{2}$, fournit le plus petit majorant de $F$ que l'on puisse obtenir avec le mécanisme considéré. On obtient ainsi une valeur par excès de $F$ et une approche par l'extérieur du convexe des chargements potentiellement supportables par l'ouvrage.

\subsection{Comparaison - approche cinématique et statique du convexe}

Dans le cas de l'ouvrage à géométrie simple repré- 


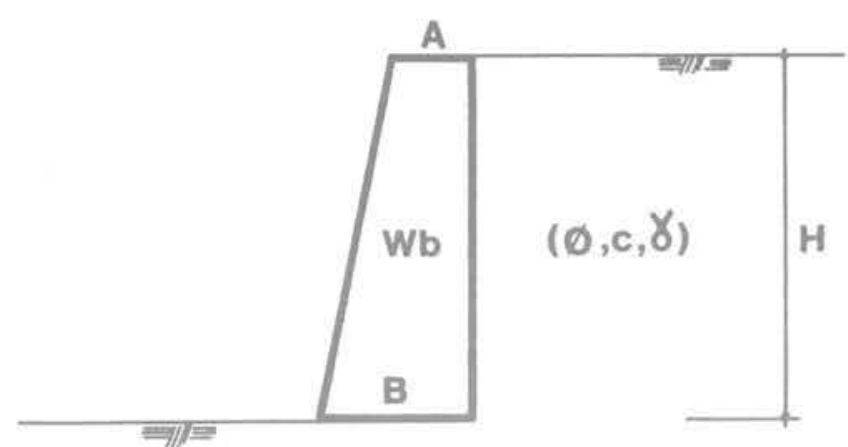

Fig. 7 L'ouvrage simple étudié (comparaison approches statique et cinématique)

senté sur la figure 7. l'approche extérieure obtenue a été comparée à une approche statique de la frontière du convexe obtenue au moyen du programme statique éléments finis de Pastor (1978) et du maillage ci-dessous (fig. 8) [discrétisation du sol en 92 triangles et 16 zones de prolongement du champ de contraintes]

La figure 9 représente les approches de la frontière du convexe des chargements potentiellement supportables ainsi déterminées pour $\phi=10^{\circ}$ et $\phi=20^{\circ}$. On constate que l'approche cinématique proposée, malgré la simplicité des hypothèses prises en compte (mur en translation, coin rigide dans le remblai) fournit des résultats corrects, compte tenu de l'incertitude sur les caractéristiques mécaniques des matériaux couramment utilisés en géotechnique $(\phi$ est rarement connue avec une précision supérieure à 2 degrès).

\subsection{Comparaison avec les méthodes classiques de calcul des murs poids}

\subsubsection{Les méthodes classiques}

On peut distinguer deux types de méthodes classiques de calcul des murs de soutènement : la méthode des trois coefficients de sécurité (sécurité au renversement, sécurité au glissement, sécurité au poinçonnement), et la méthode de la charge inclinée excentrée développée par Tran-Vo-Nhiem (1971). Ces méthodes étudient séparément l'action du remblai sur le mur et la réaction du sol de fondation. Or, on ne peut résoudre le problème de la stabilité d'un mur de soutènement en dissociant ces deux problèmes qui sont en réalité dépendants l'un de l'autre.

D'autre part, ces méthodes font intervenir simultanément des considérations d'ordre statique (contraintes admissibles par exemple) et pseudo-cinématique (étude de trois modes de rupture possibles par exemple), ce qui leur fait perdre toute signification du point de vue mécanique.

3.4.2 Comparaison des méthodes classiques avec l'approche cinématique

Dans le cas du mur poids précédent (fig. 7 ), nous avons comparé les méthodes classiques de calcul (méthode des trois coefficients, méthode de la charge inclinée excentrée) à l'approche extérieure du convexe des chargements potentiellement supportables par l'ouvrage.

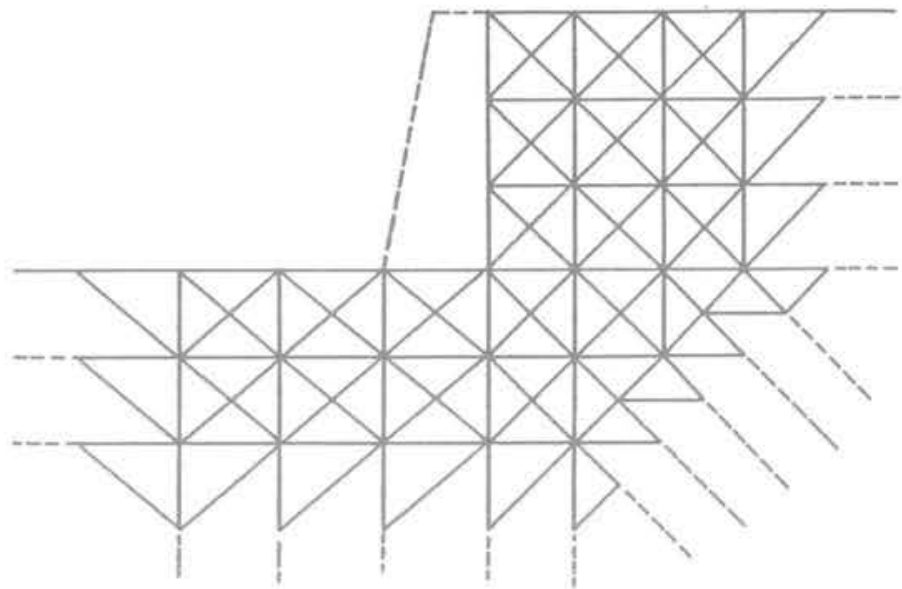

Fig. 8 Le maillage statique
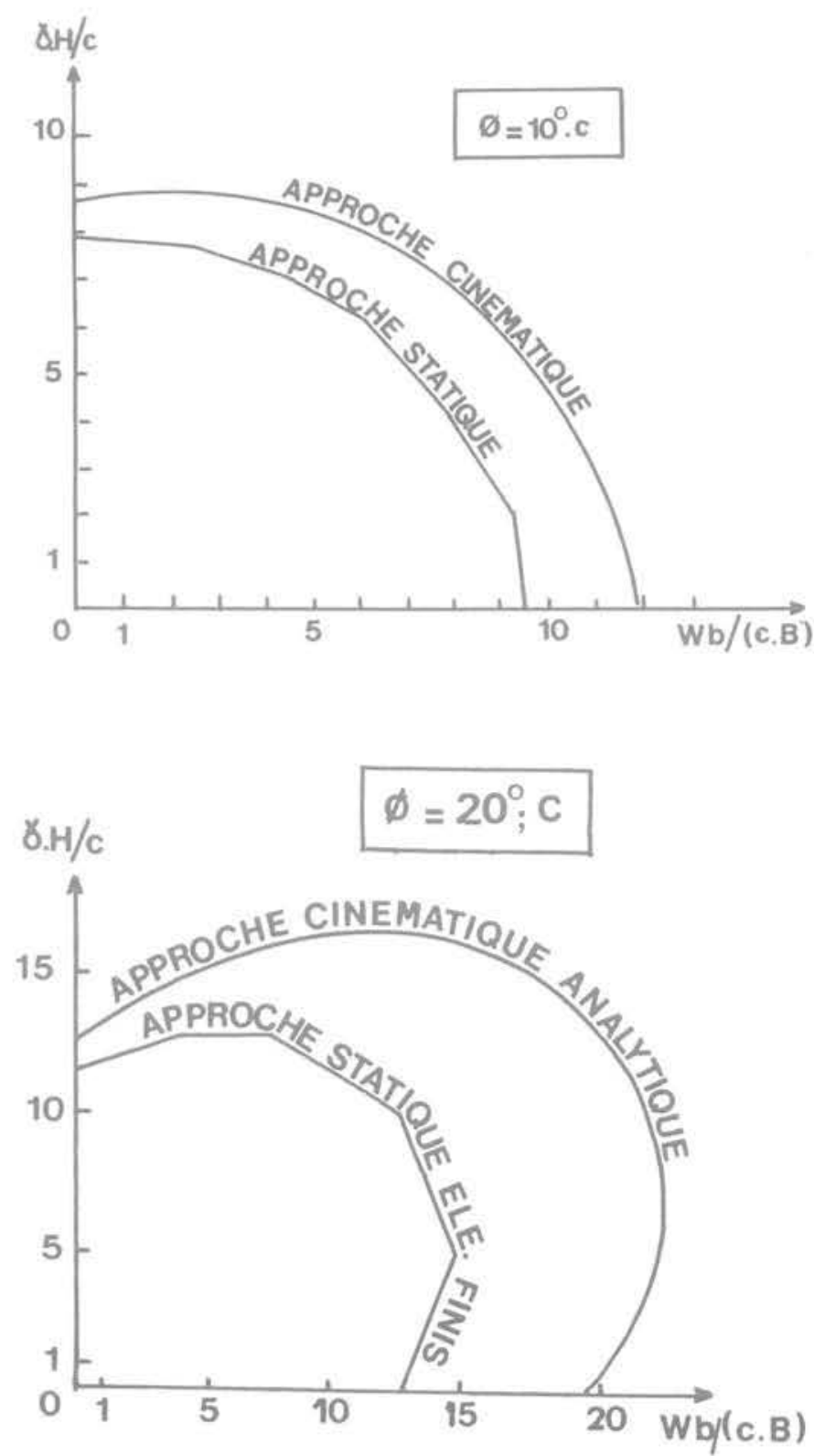

Fig. 9 Comparaison des approches statiques et cinématiques $\left(\emptyset=10^{\circ}\right.$ et $\left.20^{\circ}, A / H=0,2, B / H=0,4\right)$ 


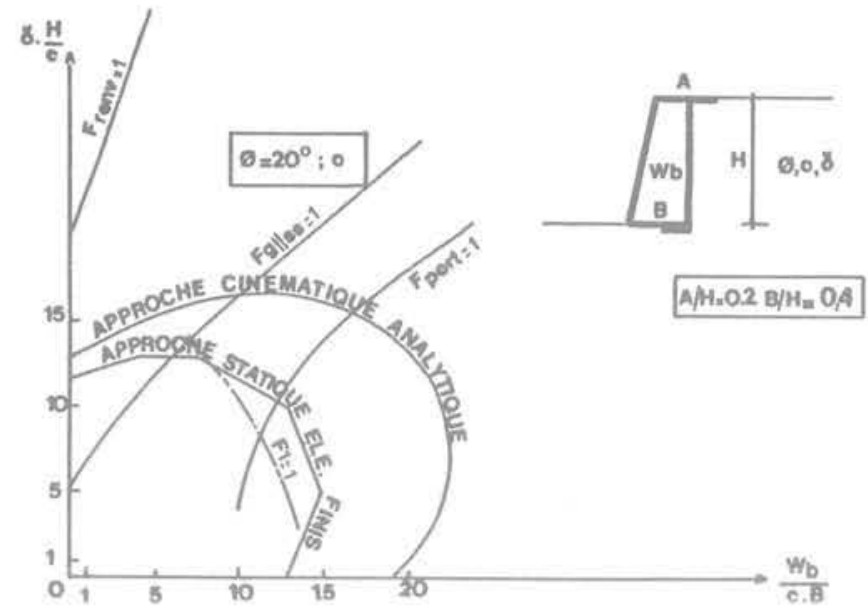

Fig. 10 Comparaison des méthodes classiques avec l'approche cinématique

La poussée qui s'exerce sur le mur est calculée à partir de la.méthode de superposition au moyen des tables de Caquot-Kerisel et Absi (1973), pour un angle d'inclinaison des contraintes $\delta=\phi$. On recherche les chargements conduisant à des coefficients de sécurité égaux ou supérieurs à l'unitè. On détermine un domaine (S) [méthode des trois coefficients] ou ( $\mathrm{S}^{\prime}$ ) [méthode de la charge inclinée excentrée] à l'intérieur duquel la méthode conclut à la stabilité du mur (fig. 10).

La méthode des trois coefficients conduit à déterminer un domaine (S) de chargement pour lequel le mur est supposé être stable; or, on peut constater (fig. 10) qu'une partie de (S) est au-delà du convexe défini par l'approche cinématique. II existe donc des chargements pour lesquels la méthode des trois coefficients conduit à considérer le mur comme stable, alors que l'on peut affirmer qu'il est instable: il ne saurait régner dans le sol un champ de contraintes respectant les conditions aux limites et les critères de résistance des matériaux et des interfaces et équilibrant de tels chargements. On constate d'autre part que le coefficient de sécurité au renversement conduit à des "chargements limites" $\left(F_{\text {renv }}=1\right)$, très éloignés de la frontière du convexe, et donc pour lesquels la rupture est certaine; ce coefficient est donc mal adapté au calcul des murs. Les valeurs des coefficients de sécurité généralement adoptées par cette méthode servent donc plus à traduire une méconnaissance du phénomène réel qu'une réelle sécurité vis-à-vis de la ruine de l'ouvrage.

La méthode de la charge inclinée excentrée conduit à déterminer une frontière en deçà de l'approche cinématique du convexe des chargements potentiellement supportables. On ne peut donc rien en conclure quant à la validité de la méthode.

\subsection{Comparaison avec les expériences}

Martial (1972) a réalisé en laboratoire des essais de stabilité de modèles réduits de murs poids de soutènement, le sol étant simulé par un matériau analogique bidimentionnel constitué de petits rouleaux en duralumin (matériau de Scheebeli - 1957)

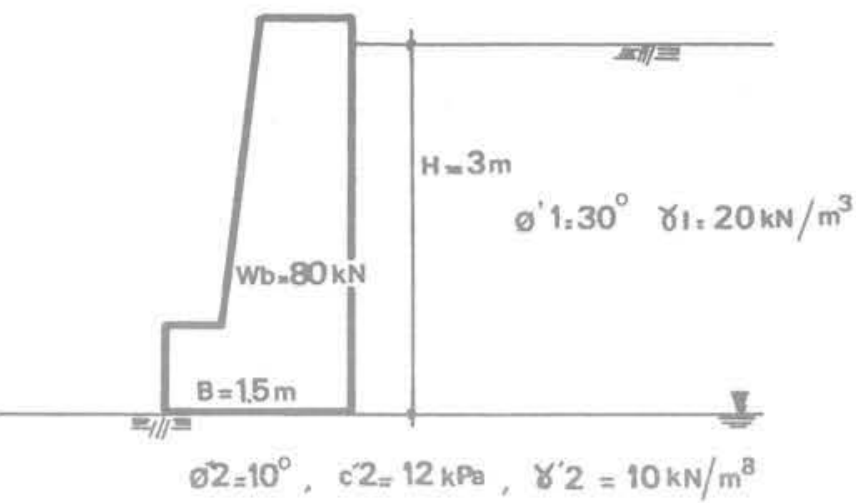

Fig. 11 Exemple d'utilisation des abaques

assimilable à un sol de Coulomb purement pulvérulent $\left(\phi=26^{\circ}, C=0, \gamma=22.5 \mathrm{kN} / \mathrm{m}^{3}\right)$. En remblayant derrière le mur par couches successives de faible épaisseur, il détermine la hauteur $\mathrm{H}_{r}$ de remblai qui provoque la rupture du mur.

Martial recherche la forme optimale des murs poids. Dans ce but, il étudie un grand nombre de murs de formes très différentes. Ces travaux l'ont amené à conclure que les murs les plus stables (à poids égal) sont ceux présentant une excentricité de la résultante sur la base du mur positive (le point de passage de la résultante est alors situé entre le remblai amont et l'axe de la fondation). La rupture se produit alors par rotation du mur vers le remblai.

Les résultats des expériences à la rupture réalisées par Martial peuvent être comparés aux solutions obtenues par le calcul à la rupture, au moyen de l'approche cinématique décrite ci-dessus. On peut ainsi calculer un coefficient $F$ tel que si $F$ est supérieur à l'unité la rupture est certaine. On obtient comme valeur moyenne de $F$ :

\begin{tabular}{l|c}
\hline $\begin{array}{l}\text { Excentricité de la } \\
\text { résultante sur la base }\end{array}$ & $\begin{array}{c}\text { Valeur moyenne de } \\
\mathrm{F}\end{array}$ \\
\hline Positive & 0,9 \\
\hline Négative & 0,6 \\
\hline
\end{tabular}

On constate donc que les murs pour lesquels l'excentricité de la rèsultante sur la base est positive conduisent à des valeurs de $\mathrm{F}$ proche de l'unité. Pour de tels murs, le mécanisme considéré semble donc bien adapté. Par contre, les murs pour lesquels l'excentricité est négative conduisent à une valeur faible de F par rapport à l'unité. II semble alors qu'il faille s'orienter vers des mécanismes permettant la prise en compte de la rotation du mur.

\section{Abaques de calcul}

\subsection{Principe}

On recherche au moyen de l'approche cinématique décrite ci-dessus une valeur approchée des coefficients multiplicateurs de charge pour un trajet de 
charge radial. On a alors :

$\mathrm{K}=\mathrm{F}_{\mathrm{i}}^{+}=\operatorname{Min}\{\mathrm{P}(\underline{\underline{\mathrm{d}})}) /(\underline{Q} \cdot \underline{q}(\underline{\mathrm{U}})) \mid \underline{\mathrm{d} C} . \mathrm{A}$. :

$$
\underline{Q} \cdot \underline{q}(\underline{U})>0\}
$$

$K$ peut se mettre sous la forme $\left(Q S_{1} \neq 0\right)$ :

$K=1 / Q S_{1} \cdot \operatorname{Min}\left\{P(\underline{d}) /\left(\underline{Q} / Q S_{1} \cdot \underline{q}(\underline{U})\right) \mid \underline{\underline{d} C} . A\right.$. :

$$
\underline{Q} \cdot \underline{q}(\underline{U})>0) \text {. }
$$

Aussi, donnons-nous des valeurs de la fonction $f$ : $\mathrm{f}=\mathrm{K} \cdot \mathrm{QS} \mathrm{S}_{1}=\operatorname{Min}\left\{\mathrm{P}(\underline{\underline{d}}) /\left(\underline{Q} / Q S_{1} \cdot \underline{q}(\underline{U})\right) \mid \underline{\underline{d}} \mathrm{C} \cdot \mathrm{A}\right.$. :

$$
\underline{Q} \cdot \underline{q}(\underline{U})>0\} \text {. }
$$

$f$ étant une fonction de $W_{b} /\left(\gamma_{1} \cdot H \cdot B\right), \gamma_{1} / \gamma_{2}^{\prime}, p_{1} /\left(\gamma_{1} \cdot H\right)$, $p_{2} /\left(\gamma_{1} \cdot H\right), \gamma_{w} / \gamma_{1}, \phi_{1}^{\prime}, \phi_{2}^{\prime}, C_{1}^{\prime} / C_{2}^{\prime}, B / H, H_{w} / H, \alpha, \beta, \lambda$. Pour se ramener au coefficient $K$, il suffit de diviser $f$ par

$$
\mathrm{QS}_{1}=\gamma_{1} \cdot \mathrm{H} / \mathrm{C}_{2}^{\prime} \text {. }
$$

\subsection{Exemple d'utilisation}

Considérons le mur représenté sur la figure 11.

On a :

$$
\alpha=0, \quad \beta=0, \quad \lambda=0, \quad B / H=0,5,
$$

$\mathrm{H}_{w} / \mathrm{H}=0, \quad \gamma_{1} \cdot \mathrm{H}^{\prime} \mathrm{C}_{2}^{\prime}=5, \quad \gamma_{2}^{\prime} / \gamma_{1}=0,5, \quad \phi_{1}^{\prime}=30^{\circ}$.

$\phi_{2}^{\prime}=10^{\circ}, \quad C_{1}^{\prime} / C_{2}^{\prime}=0, \quad W_{b} /\left(\gamma_{1} \cdot H \cdot B\right)=0,89$.
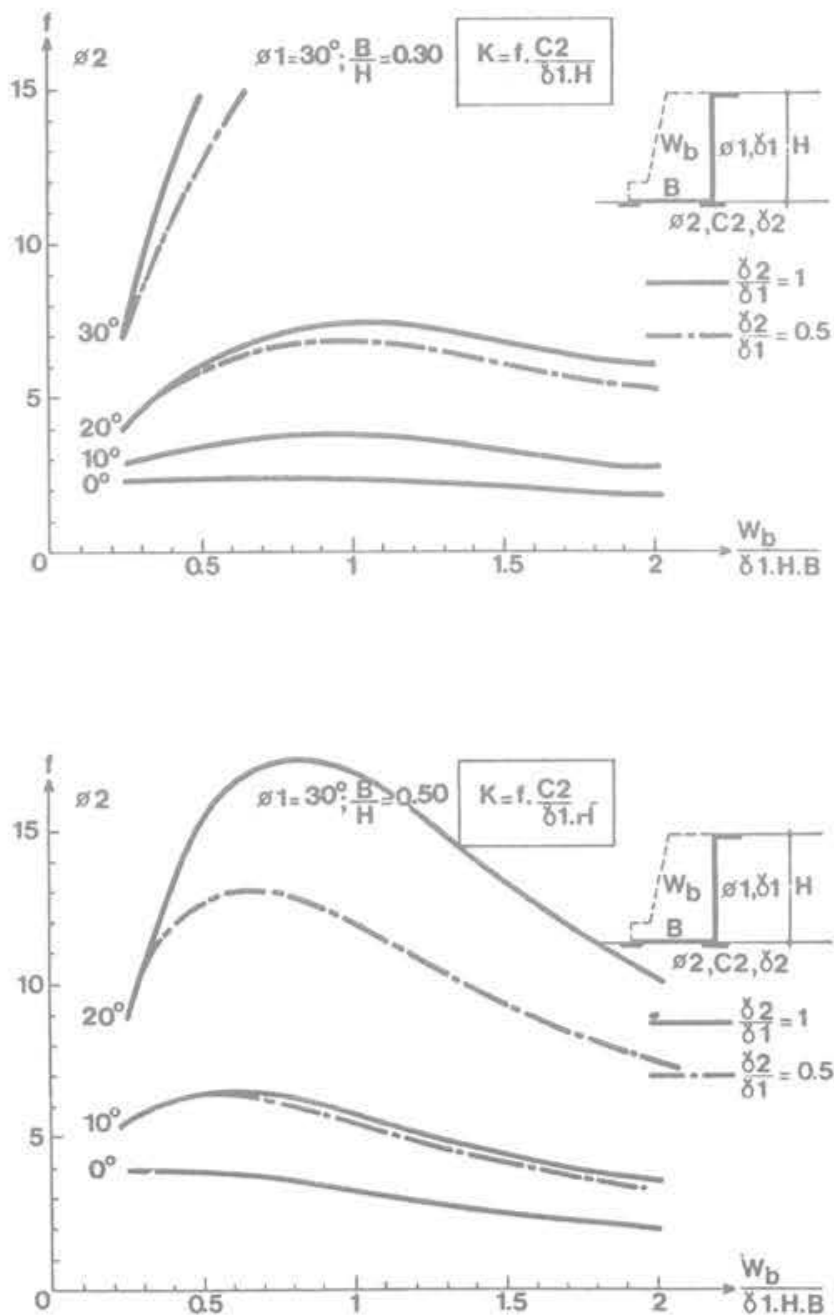

En se reportant aux abaques ci-dessous, on obtient $f=5,7$, soit

$$
K=f /\left(\gamma_{1} \cdot H / C_{2}^{\prime}\right)=1.14 \text {. }
$$

On peut donc affirmer que si la cohésion du sol d'assise diminue de $C_{2}^{\prime}=12 \mathrm{kPa}$ à $\mathrm{C}_{2}^{\prime}=10.5 \mathrm{kPa}$, le mur devient instable. De même, on peut voir, en se reportant à l'abaque correspondant à $\phi_{1}^{\prime}=30^{\circ}, \phi_{2}^{\prime}=0^{\circ}$, pour lequel $f=3,4$ (soit $K=0,7$ ), que si l'angle de frottement du sol d'assise a été surestimé, le mur devient instable.

\subsection{Abaques}

Nous donnons ci-dessous des abaques dans le cas:

$$
H_{w}=0, \quad \alpha=0, \quad \beta=0, \quad \lambda=0, \quad p_{1}=0, \quad p_{2}=0
$$

et pour des valeurs de $\gamma_{1} / \gamma_{2}^{\prime}$ égales à 0,5 et $1, \phi_{1}^{\prime}$ compris entre $30^{\circ}$ et $40^{\circ}$, $\phi_{2}^{\prime}$ compris entre $0^{\circ}$ et $30^{\circ}$. Dans des cas géométriques ou mécaniques plus complexes, ou si l'on désire étudier d'autres trajets de charge, le programme de calcul donné dans Mommessin (1981) permet d'obtenir la valeur minimale de $K$ et des coefficients multiplicateurs de charge associés.
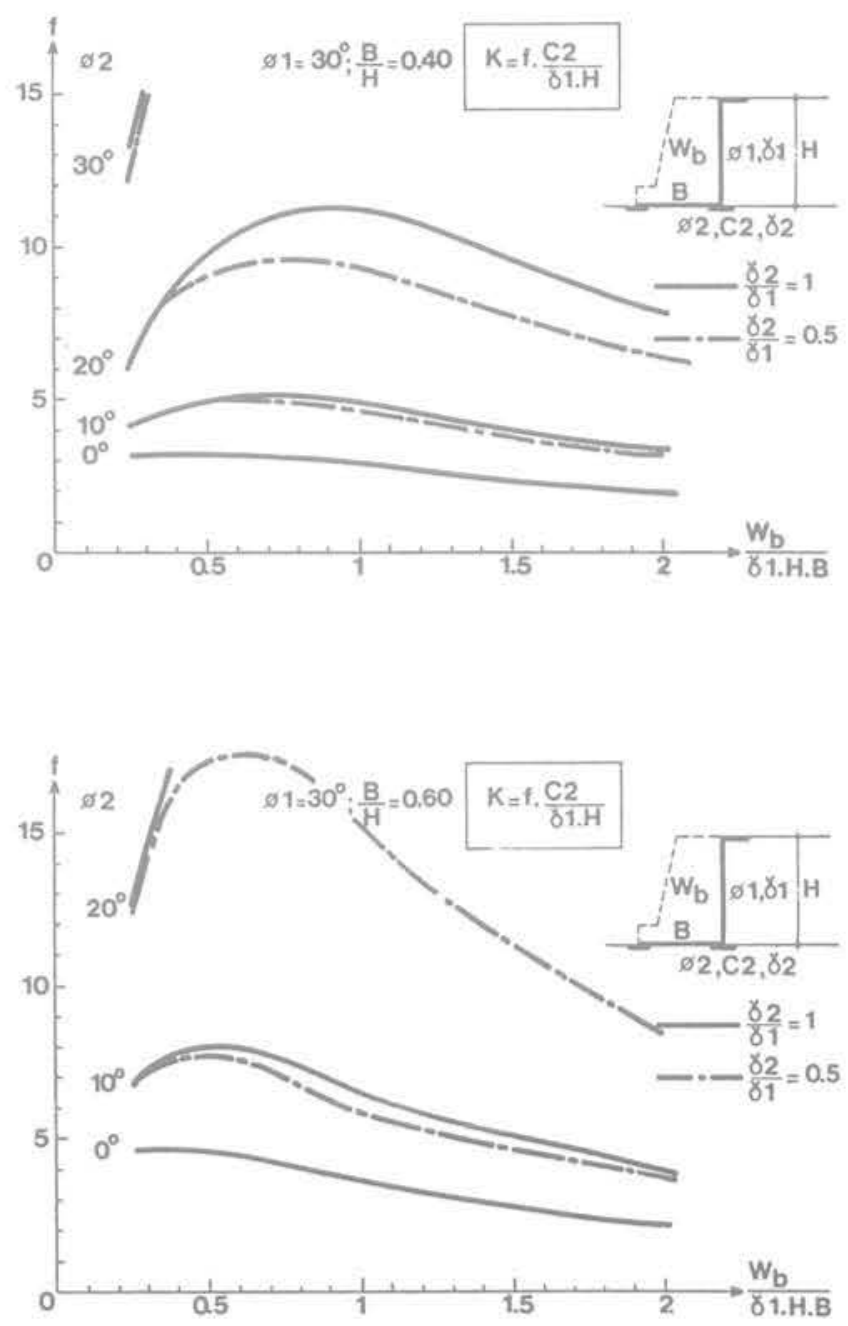

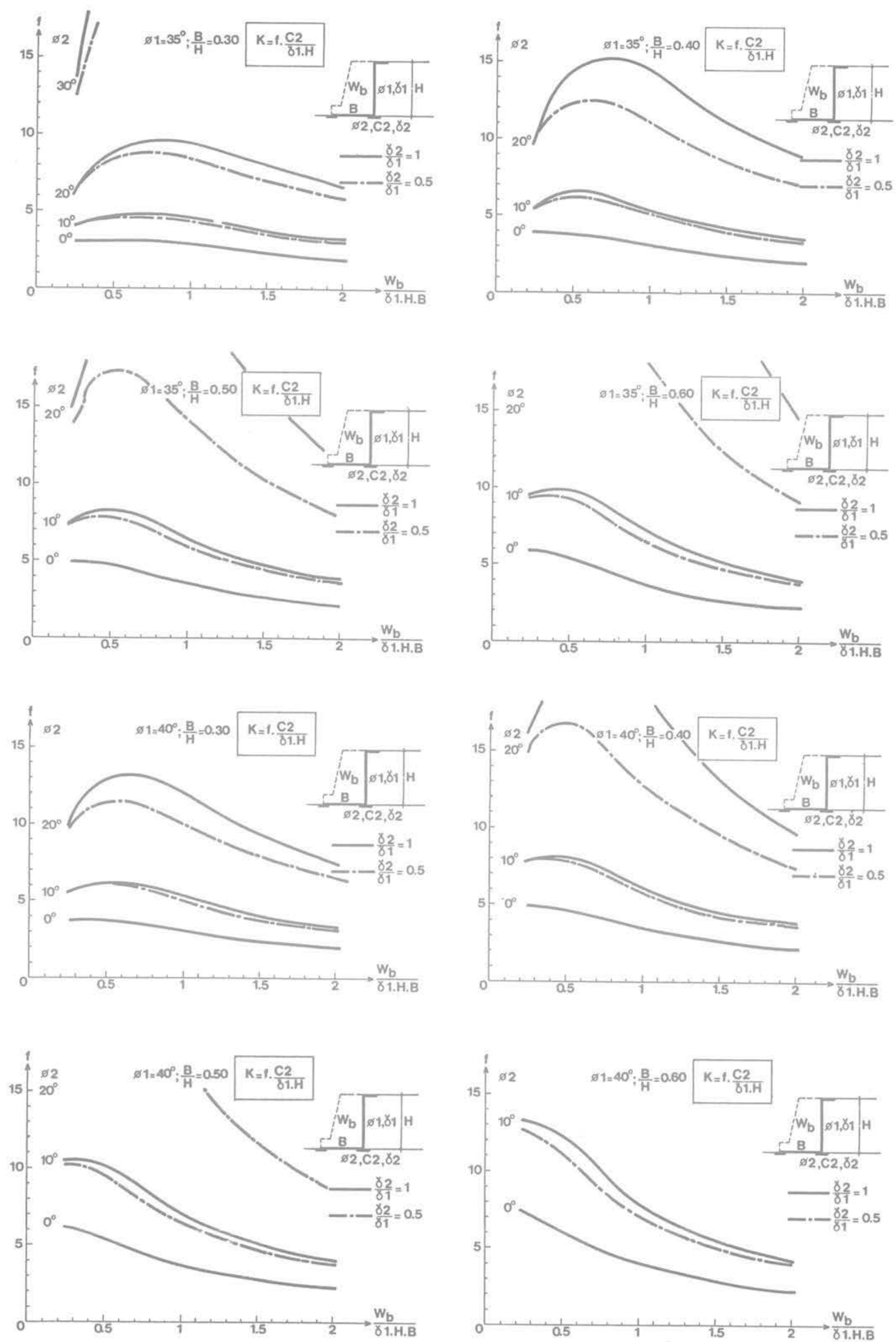


\section{Conclusions}

L'application du calcul à la rupture à l'étude de la stabilité des murs poids de soutènement permet une meilleure interprétation des méthodes classiques de calcul des murs : ces méthodes n'ont pas de signification mécanique claire et peuvent parfois conclure à la stabilité de l'ouvrage alors que l'on est dans le domaine de la ruine certaine.

Le mécanisme proposé fournit une approche cinèmatique simple du problème étudié. La comparaison avec les expériences sur modèle réduit de murs montre que cette approche fournit de bons résultats lorsque l'excentricité de la résultante sur la base du mur est positive.

La définition d'un accroissement ultime de charge pour lequel la rupture de l'ouvrage est certaine constitue une méthode rigoureuse de vérification de la stabilité des ouvrages; il permet d'autre part de connaître l'influence des divers paramètres de chargements sur la stabilité de l'ouvrage.

En ce qui concerne l'utilisation des résultats obtenus pour le calcul des murs de soutenement, il convient d'insister sur le fait que la méthode proposée ne peut constituer qu'une méthode de vérification: en 'absence d'hypothèses supplémentaires (loi de comportement) on ne peut jamais affirmer la stabilité de l'ouvrage. Seule sa rupture peut être déterminée de façon certaine.

Les auteurs tiennent à remercier Monsieur J. Pastor pour l'aide qu'il leur a apporté pour la formulation du problème du mur de soutènement sur le plan numérique et pour les modifications du programme statique qui en ont résulté.

\section{Références bibliographiques}

Caquot A., Kerisel J. et Absi E. (1973)

"Tables de poussée et de butée." Gauthier-Villars Paris.
Coussy O. (1978)

"Le calcul à la rupture dans la tenue des ouvrages en terre. " Thèse de Docteur-Ingénieur - Université de Paris VI.

Drucker D.C. (1953)

"Limit analysis of two and three dimensional soil mechanics problems, $m$

Jnl. of the Mech. and Phys. of Sol., vol. 1, $n^{\circ} 4$.

Martial G. (1972)

«Action des terres sur les ouvrages de soutènement. Stabilité des murs poids, "

Thèse de Docteur-Ingénieur - Université de Grenoble 1.

Mommessin M. (1981)

"Application du calcul à la rupture à l'étude de la stabilité des murs poids de soutènement. m

Thèse de Docteur-Ingénieur - Université de Grenoble 1.

Pastor J. (1978)

"Analyse limite : détermination numérique des solutions statiques complètes. Application au talus vertical. $m$

Journal de Mécanique Appliquée, vol. 2, n². pp. 167196.

Radenkovic (1961)

"Théorèmes limites pour un matériau de Coulomb à dilatation non standardisée. $m$

CR. AC. SC. Paris 252, pp. 4103-4104.

Salençon J. (1978)

"Caicul à la rupture et analyse limite."

Cours E.N.P.C. - Paris.

Scheebeli (1957)

"Une analogie mécanique pour l'étude de la stabilité des ouvrages en terre à deux dimensions." $4^{\text {th }}$ I. C. S. M. F. E. - vol. II, pp. 228-232 - Londres.

Tran-Vo-Nhiem (1971)

* Force portante limite des fondations superficielles et résistance maximale à l'arrachement des ancrages, $x$

Thése de Docteur-Ingénieur - Faculté des Sciences de Grenoble. 
